\title{
Percepção da dor musculoesquelética relacionada ao uso excessivo de smartphone e notebook por estudantes de graduação
}

O desenvolvimento tecnológico tem como objetivo facilitar a vida do ser humano, economizando seu tempo e tornando mais fácil o acesso à informação e a comunicação. Porém, o uso excessivo destas, por mais de 2 a 4 horas diárias, associado a posições inapropriadas no período de uso, acumulam lentamente prejuízos para o corpo resultando no aparecimento de dores no sistema musculoesquelético. $O$ objetivo é analisar a percepção da dor e desconforto no sistema musculoesquelético frente ao uso excessivo de smartphone e notebook em estudantes de graduação. A pesquisa foi quantitativa descritiva transversal, sendo selecionados para este estudo, 37 estudantes do Curso de Fisioterapia de ambos os gêneros com média de idade de 21 anos. Para a realização da coleta dos dados foram utilizados, o questionário Mil, a escala visual analógica (EVA), questionário de Usuários de Tecnologia, e o questionário de Dickinson. A prevalência de tempo de uso diário do smartphone foi de 5 a 6 horas, em relação ao notebook de 2 a 4 horas diariamente, permanecendo maior parte do dia conectada a estas tecnologias, resultando em dores em diversas partes do corpo, principalmente na coluna cervical, ombros e coluna lombar. 0 estudo demonstrou alta prevalência (76\%) de dor musculoesquelética nos entrevistados, sendo assim, é necessária a conscientização do uso destas tecnologias, a fim de prevenir índices de queixas relacionadas ao uso indiscriminado.

\section{Perception of musculoskeletal pain related to excessive use of smartphone and notebook by undergraduate students}

Technological advance aims to make life easier for the human being, saving time and making access to information and communication easier. However, excessive use of these, for more than 2 to 4 hours daily, associated with inappropriate positions in the period of use, slowly accumulate damages to the body, resulting in the appearance of pain in the musculoskeletal system. The objective is analyze the perception of pain and discomfort in the musculoskeletal system against the excessive use of smartphone and notebook in undergraduate students. Quantitative cross descriptive, being selected for this study, 37 students of the Physical Therapy Course, average of 21 years. In order to perform the data collection, there were made the MCGill questionnaire on the visual analog scale (VAS), Questionnaire of Technology Users, and the questionnaire of Dickinson. The prevalence of smartphone use time was from 5 to 6 hours, compared to the notebook which was from 2 to 4 hours daily, remaining most of the day connected to these technologies, and resulting in pain in various parts of the body, especially in the cervical spine, shoulders and lumbar spine. The study showed a high prevalence (76\%) of musculoskeletal pain in the interviewees, so it is necessary to increase awareness of the use of these technologies in order to prevent complaints related to the indiscriminate use.

Keywords: Muscle pain; Undergraduate Students; Cellphone; Impulse control disorders.

Topic: Fisioterapia

Reviewed anonymously in the process of blind peer.

\section{Vinícius Brandalise}

Universidade Comunitária da região de Chapecó, Brasil

http://lattes.cnpq.br/5368531161314164

\section{viniciusbrandalise@gmail.com}

\section{Anelise Binsfeld}

Universidade Comunitária da região de Chapecó, Brasi

anelisebinsfeld@unochapeco.edu.br

Mariane Zanetti

Universidade Comunitária da região de Chapecó, Brasil marianezanetti@unochapeco.edu.br
Received: 10/02/2021

Approved: 20/03/2021

\section{Ricardo Lazarotto \\ Universidade Comunitária da região de Chapecó, Brasil \\ http://lattes.cnpq.br/3540052815768228 \\ http://orcid.org/0000-0002-7753-6239}

ricardolazarotto@gmail.com
Referencing this:

BRANDALISE, V.; BINSFELD, A.; ZANETTI, M.; LAZAROTTO, R.

Percepção da dor musculoesquelética relacionada ao uso excessivo de smartphone e notebook por estudantes de graduação. Scire Salutis, v.11, n.2, p.51-62, 2021. DOI: http://doi.org/10.6008/CBPC22369600.2021 .002 .0006 


\section{INTRODUÇÃO}

O desenvolvimento tecnológico no decorrer das últimas décadas tem alterado a forma do ser humano se relacionar em sociedade e com o mundo; pois, o homem procurou desenvolver técnicas que facilitassem sua vida em sociedade, e um dos pontos principais para a melhoria desta, foi desenvolver a inclusão digital, com o objetivo de facilitar a vida do ser humano, economizando seu tempo e tornando mais fácil o acesso à informação e a comunicação, contribuindo para a evolução da humanidade (TAPSCOTT, 2010).

Entretanto com o fortalecimento da internet como ferramenta cotidiana, antigos comportamentos e costumes vêm sofrendo alterações. Hoje, por exemplo, a comunicação é muito mais frequente pelos dispositivos e pela internet, que permite a participação em uma ampla gama de atividades, que vão muito além do tradicional oral e comunicação escrita entre indivíduos. Esses costumes estão cada vez mais distantes da população jovem e, com isso, vem principalmente do fortalecimento de ambientes virtuais de informação e das redes sociais de comunicação (SOUZA et al., 2014).

Com a facilidade ao acesso a esses dispositivos eletrônicos, o tempo gasto com os aparelhos eletrônicos tem aumentado e tem gerado uma dependência dos mesmos. O que muitas pessoas não sabem, é que, o uso excessivo deles pode causar danos ao corpo humano, relacionados tanto ao tempo, quanto à postura e, decorrente disso, consequências negativas à saúde do público jovem/adulto têm sido descritas na literatura, tais como alterações no sono, cansaço, ansiedade, depressão, excesso de peso, redução nos níveis de atividade física, diminuição da concentração, surgimento de dores musculoesqueléticas, estresse e cefaleia (SAUERESSIG et al., 2015).

A falta de pausas durante o uso dessas tecnologias, somada ao esforço excessivo de atividades, posições impróprias do corpo e longos períodos de utilização, acumulam lentamente prejuízos para o corpo e podem provocar lesões em várias regiões, principalmente nos membros superiores, tais como mãos, punhos, braços, ombros, pescoço, cabeça e a coluna vertebral, as quais acabam se transformando em uma condição grave e muito dolorosa que não se apresenta de imediato. São dores que vêm se somando ao longo do tempo, aumentam o risco de desenvolvimento, caso o usuário utilize-as por muitas horas seguidas e diariamente (COOPERA et al., 2009).

Dentre os fatores citados anteriormente, destaca-se o aparecimento de dores musculoesqueléticas, pois, a alteração do fuso muscular principalmente do estiramento deste, leva ao aparecimento de pontos miofasciais e contraturas, levando consequentemente ao aumento da tensão dessa musculatura que no presente estudo em maior referência trata-se de músculos posteriores da região cervical sendo um fator predisponente ao aparecimento de cefaleias tensionais, além disso, a contratura muscular não se dissolve durante o relaxamento o que poderá interferir na qualidade do sono e de repouso dentre outros fatores acima descritos (MAGEE, 2005).

Em grande parte, as alterações musculoesqueléticas estão relacionadas com a vida moderna, na qual as pessoas passam grande parte do seu tempo sentadas; o excesso de tempo traz consequências negativas à saúde e à qualidade de vida, decorrente do nível de concentração que as tarefas da vida 
moderna exigem (TEIXEIRA et al., 2006) e, essas posturas vêm se tornado comuns no dia a dia das pessoas, tanto no trabalho, em casa, ou no lazer (ANDREGHETTO, 2015).

O estabelecimento da má postura com o uso excessivo diário de dispositivos eletrônicos, segundo Saueressig et al. (2015) estabelece que, o uso por tempo maior que três horas por dia, pode gerar contraturas musculares, resultando na dor. Normalmente, os usuários utilizam o dispositivo com uma ou as duas mãos abaixo da altura dos olhos, essa postura leva cada vez mais ao aumento da flexão da coluna cervical, resultando em tensão muscular e a perda da curvatura normal (GUAN et al., 2016).

Estudos anteriores afirmam que o peso suportado pela coluna cervical aumenta dramaticamente quando flexionada em graus variados. Uma cabeça de adulto pesa, em média, 4,53 kg na posição neutra, à medida que a cabeça se anterioriza aumenta o peso e grau de inclinação, ou seja, quanto maior a inclinação maior será o peso suportado pela cervical (PARK et al., 2015).

Outra frequente causa de alteração na postura da cabeça e do pescoço é a falha nos músculos posteriores assim, a curvatura normal é perdida ou invertida. O mau alinhamento dos ombros também é uma alteração frequente, provocando desvios posturais na coluna vertebral, principalmente na coluna cervical devido à flexão excessiva. Esses fatores levam a sobrecarga e o aparecimento de pontos dolorosos (HOFFMANN et al., 2009).

Considerando as mudanças dos processos lesivos ao homem, a fisioterapia enquanto ciência que estuda o movimento humano buscou compreender os mecanismos biomecânicos que interferem na saúde do indivíduo moderno, assim o presente estudo propõe analisar a percepção dolorosa frente ao uso excessivo de smartphone e notebook em estudantes de graduação.

\section{METODOLOGIA}

Trata-se de estudo quantitativo, descritivo de corte transversal, realizado na Universidade Comunitária da Região de Chapecó - UNOCHAPECÓ. A realização da pesquisa se deu após a aprovação do projeto pelo Comitê de Ética e Pesquisa (CEP), mediante ao no de protocolo 1.764 .867 e após assinatura do Termo de Consentimento Livre e Esclarecido pelos estudantes, a coleta de dados aconteceu no mês de outubro de 2016.

A população foi composta por 37 estudantes do curso de Fisioterapia do 2o ao 8o período. A amostra foi realizada de forma aleatória intencional para ambos os gêneros.

Adotaram-se os seguintes critérios de inclusão: estudantes maiores de idade (18 anos), onde os mesmos deveriam estar devidamente matriculados no curso de fisioterapia a partir do 2 o período. Foram excluídos da amostra estudantes menores de idade, e preenchimento inadequado dos questionários.

Para a realização da coleta de dados foram aplicados quatro questionários: na qual o questionário adaptado de Lucena et al. (2015); Saueressing et al. (2015), usuários de tecnologias continha questões relacionadas ao uso excessivo das tecnologias (smartphones e notebooks) elencando questões quanto ao tempo da utilização das tecnologias, dores advindas após o uso, qual período do dia é mais utiliza as tecnologias. 
Questionário de MCGill com objetivo de identificar a localização da dor, a caracterização da dor através das palavras opcionais, como a dor se altera no decorrer do tempo e sua intensidade (PIMENTA et al., 1996).

Escala Visual Analógica (EVA): Instrumento unidimensional para a avaliação da intensidade da dor. Trata-se de uma linha com as extremidades numeradas de 0-10. Em uma extremidade da linha é marcada "nenhuma dor" e na outra "pior dor imaginável" (MARTINEZ et al., 2011).

Questionário de Dickinson et al. (1992), que consiste em perguntas relacionadas quanto a dor, desconforto nos últimos 12 meses, se há interferência da dor/desconforto nos últimos 7 dias, e se durante os últimos 12 meses foi impedido de realizar as AVD's.

Primeiramente foi realizado contato com o professor responsável pela aula no dia da coleta para receber a autorização para sua execução. Posteriormente foi realizado o convite formal, após a aceitação foi dada sequência para efetivação da assinatura do termo de consentimento livre esclarecido por parte dos participantes e em seguida a aplicação dos questionários.

Os estudantes foram informados, que para aqueles que não apresentavam dor não efetuarem o preenchimento do questionário de MCGill, escala de EVA e questionário de Dickinson et al. (1992), nesses casos será repassado somente o questionário dos Usuários de Tecnologias.

O processo de análise dos dados foi efetuado por meio do Excel For Windows Versão 2016; para a descrição, foram utilizadas as medidas descritivas de média e desvio-padrão para os dados paramétricos.

\section{RESULTADOS}

Participaram do estudo 37 estudantes, com idade média de 21 anos (DP: \pm 5.08 ), com idade mínima de 18 anos e máxima de 49 anos; sendo 86,48\% ( $n=32$ ) do gênero feminino e 13,51\% ( $n=5)$ do gênero masculino, a renda média familiar foi de 4 a 8 salários mínimos, que representam $32 \%$ ( $n=12)$.

A determinação do tempo de uso deu-se a partir da medida do tempo médio diário (horas) despendido na utilização de notebook e smartphone, na qual os resultados apontam que $59 \%(n=22)$ dos estudantes utilizam o notebook de 2 a 4 horas diariamente, e 35\% (n=13) utilizam smartphone de 5 a 6 horas diariamente, conforme mostra a figura 1.

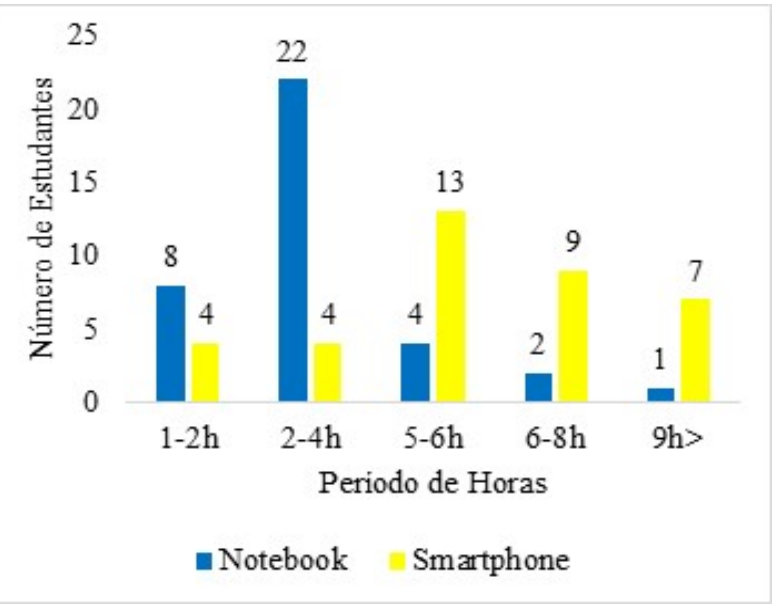

Figura 1: Relação do uso diário pelos estudantes. 
Quanto ao período do dia que mais utilizam as tecnologias observou-se que o período noturno tem maior predominância com $n=36$, já no período vespertino $\operatorname{com} n=13$, e no período matutino com $n=5$, notase que nessa questão a maioria dos questionários apresentava mais de uma opção assinalada.

Ao analisar o uso do smartphone e notebook, $n=31$ dos participantes utilizam como ferramenta de trabalho, em vista que $n=31$ utilizam notebook e os $n=6$ smartphone. Segundo os resultados $n=10$ utilizam para fins de conexão com redes sociais, $n=2$ utilizam para outros fins.

Em relação ao esquecimento de realizar as tarefas quando estão utilizando alguma das tecnologias, constatou-se que $24 \%(n=7)$ dos estudantes esquecem de realizar as atividades.

Quanto ao tempo de uso (anos) das tecnologias (smartphone e notebook) nota-se que $38 \%(n=14)$ utilizam o notebook há mais de 7 anos, e 41\% ( $n=15)$ o utilizam o smartphone há mais de 7 anos, conforme mostra a figura 2.

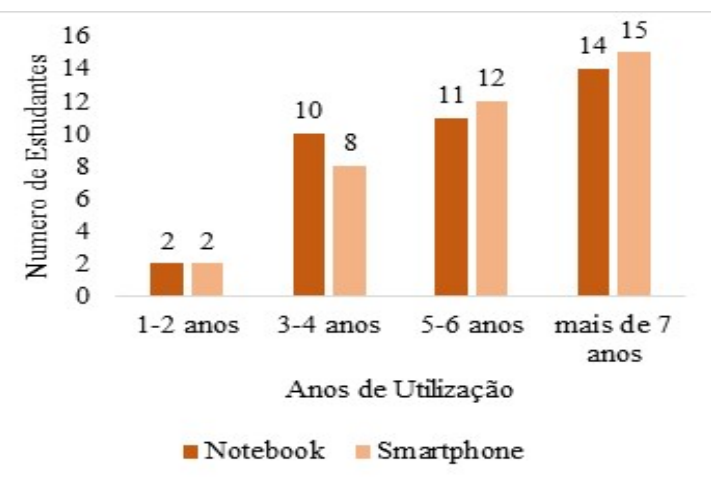

Figura 2: Relação do tempo total do smartphone e notebook.

Com relação às diferentes regiões anatômicas e sintomas dolorosos, entre os estudantes entrevistados, $n=28$ que apresentaram dor ou desconforto nos últimos doze meses. A região que apresentou maior prevalência de queixas dolorosas foi a coluna cervical, $n=19$ mencionaram dor na região, seguida de dor em ombros resultou em $n=5$ referem dor em ombro direito, $n=e m$ ombro esquerdo, $n=6$ em ambos os ombros, $n=2$ apresentaram dor em cotovelo direito e $n=1$ em ambos os cotovelos, que apresentaram dor/desconto em punho/mão direita resultando em $n=10$ ambos punhos/mãos $n=4$. Na região lombar $n=15$ apresentam dor na região.

Quanto a dor ou desconforto nos últimos sete dias n=14 dos estudantes referem dor, nisso, $n=10$ apresentam dor/desconforto na coluna cervical. Em ombro esquerdo $n=2$ relatam dor/desconforto já em ambos os ombros $\mathrm{n}=15$ apresentam dor/desconforto. Na região de cotovelos $\mathrm{n}=1$ apresentam dor/desconforto em cotovelo esquerdo. Em punho/mão direita $n=2$ apresentam dor/desconforto, em ambos punhos/mãos $n=1$, em relação a dor na coluna lombar $n=8$ apresentam dor/desconforto nos últimos 7 dias, conforme tabela 1 abaixo:

Em relação ao impedimento de realizar as atividades de vida diária (AVD's) nos últimos 12 meses, apenas $\mathrm{n}=2$ apontaram que a dor/desconforto interferiram nas AVD's. Quanto as características das dores musculoesqueléticas, avaliado através do questionário MCGill, obteve-se uma média de 15,08 pontos (DP: $\pm 8,35$ ), máxima de 20 pontos e mínimo 0 . Em relação ao índice da dor obteve-se uma média de 26,9 pontos (DP: \pm 15.66 ) com máxima de 47 pontos e mínima de 0, conforme mostra a figura 3 . 
Tabela 1: Prevalência da dor de acordo com a região.

\begin{tabular}{lll}
\hline Região & Dor/Desconforto nos últimos 12 Meses & Dor/Desconforto últimos 7 Dias \\
\hline Dor ou Desconforto & $76 \%(n=28)$ & $38 \%(n=14)$ \\
Cervical & $51 \%(n=19)$ & $27 \%(n=10)$ \\
Ombro D & $14 \%(n=5)$ & 0 \\
Ombro E & $11 \%(n=4)$ & $5 \%(n=2)$ \\
Ambos os ombros & $16 \%(n=6)$ & $14 \%(n=15)$ \\
Cotovelo D & $5 \%(n=2)$ & 0 \\
Ambos Cotovelos & $3 \%(n=1)$ & $3 \%(n=1)$ \\
Punho/Mão Direita & $27 \%(n=10)$ & $5 \%(n=2)$ \\
Ambos punhos/mãos & $11 \%(n=4)$ & $3 \%(n=1)$ \\
Lombar & $41 \%(n=15)$ & $22 \%(n=8)$ \\
\hline
\end{tabular}

Legenda: $\mathrm{n}$ : número da amostra; \% percentual da amostra.

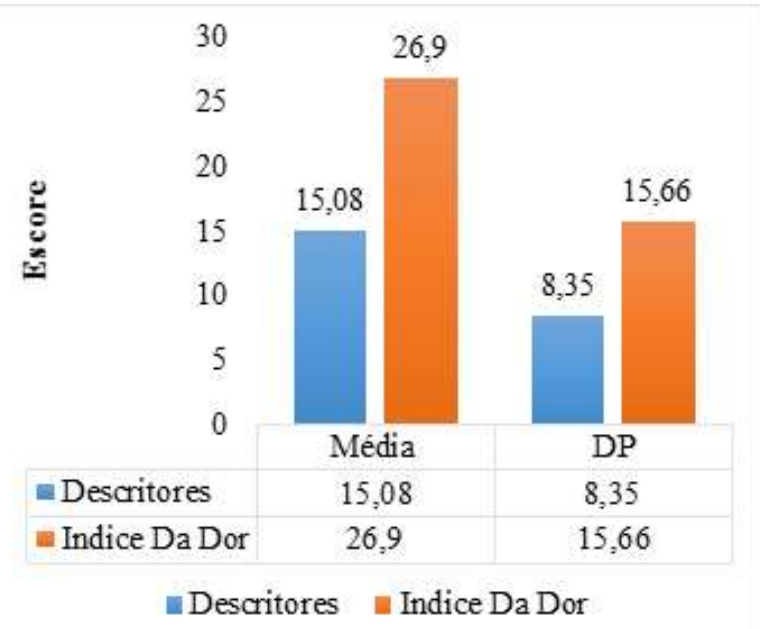

Figura 3: Análise do questionário MCGill, apontando os descritores e a índice da dor.

Foram elencadas questões quanto ao aumento da dor como também a diminuição dela. Nos resultados obtidos, observa-se que $n=25$, referem dor se permanecer em uma mesma postura em demasia, n=18 ao realizar movimentos repetitivos, $n=7$ não apresentam dor. Em relação ao alívio da dor, $n=15$ relatam melhora com alongamentos e caminhadas, $n=13$ com repouso, $n=21$ com terapia manual e relaxante muscular, outros e sem dor $n=7$.

Quanto a intensidade da dor, $22 \%(n=10)$ referem dor 3 classificada como dor leve, $18 \%(n=8)$ referem dor 10 classificada como grave, $16 \%(n=7)$ referem dor 5 classificada como moderada, conforme figura 4.

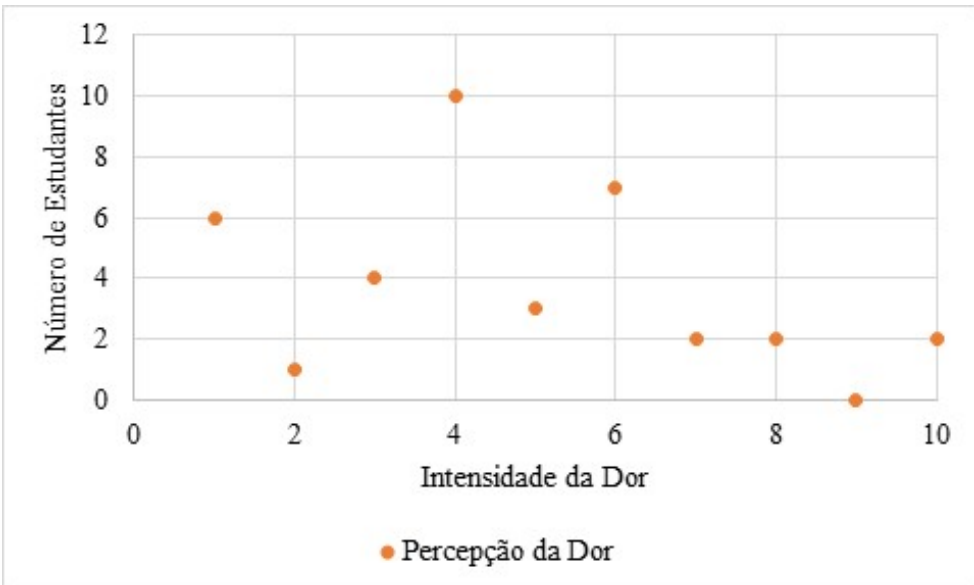

Figura 4: Análise da dispersão da percepção da dor conforme EVA.

Quanto à relação da percepção da dor e o uso de horas diárias, observa-se que quanto maior o 
tempo de uso diário maior a incidência de dores musculoesqueléticas.

\section{DISCUSSÃO}

A vida digitalizada e hiperconectada do século XXI é resultado de crescentes avanços tecnológicos, notadamente na área da comunicação, devido à conjugação da telefonia móvel com a rede mundial de computadores, capazes de permitir a interação social e a integração de conhecimentos. Acredita-se que a sociedade contemporânea passa por transformações tecnológicas, alterando de forma significativa a maneira como as pessoas se relacionam e interagem uma com as outras (PICON et al., 2015).

Os resultados obtidos neste estudo apontam que 59\% ( $n=22)$ dos estudantes utilizam notebook de 2 a 4 horas diariamente, e 35\% ( $n=13)$ utilizam smartphone de 5 a 6 horas diariamente. Assim, os resultados obtidos no estudo se assemelham com as informações da Anatel (TELECO, 2016), na qual apontam que o brasileiro passa, por dia, 3 horas e 46 minutos conectado à internet pelo celular, e mais 5 horas e 26 minutos, conectado via computador ou tablet. Em outro estudo conduzido por Young et al., (1998), observaram que o uso médio relatado pelos usuários foi de 4 a 10 horas por dia, aumentando para 10 a 14 horas nos finais de semana, isto representa em torno de 40 a 78 horas por semana de uso excessivo de internet. Com isso, pode-se inferir que é alto o tempo despendido com o uso de notebook e smartphone no tempo total de uso encontrado entre os estudantes avaliados no nosso estudo. Nesse sentido, a carga horária excessiva no uso desses equipamentos, por parte dos estudantes, possivelmente se deve ao aumento das atividades que são possíveis de serem realizadas por meio dos dispositivos eletrônicos, bem como à maior disponibilidade desses equipamentos nos mais diversos locais (KING et al., 2011).

Quanto ao tempo de uso (anos) das tecnologias (smartphone e notebook) nota-se que $38 \%(n=14)$ correspondem ao notebook e 41\% ( $n=15)$ correspondem ao smartphone, utilizam estas a mais de 7 anos; relacionando os dados com a média de idade de 21 anos (DP: \pm 5.08 ), observa-se que um terço dos estudantes passaram a maior parte da sua vida conectados a estas tecnologias. Este estudo corrobora com os achados, realizado por Pontes e Patrão (PONTES et al., 2014), na qual, os autores observaram que a utilização da internet reportada pelos jovens/adultos, com média de 21 anos resultou em uma média de 13 anos utilizando essas tecnologias. Para Khan et al. (2012) e Das (2014), estes recursos estão sendo utilizados de forma exagerada e inadequada, o que de fato vem sendo um fator preocupante para a saúde desses usuários.

Podemos entender que o uso do aparelho smartphones e de notebooks, especialmente em relação à população jovem, esteja relacionado aos aspectos de inclusão social e conectividade entre os amigos. Há pessoas que não conseguem ficar sem o celular ou notebook nem por um instante. Essas pessoas entram num estado de profunda ansiedade e angústia quando se veem sem os aparelhos, quando ficam sem conectividade ou quando a bateria se encontra no fim. A necessidade de estar conectado ultrapassa os limites e esse hábito pode representar um grande perigo para a saúde emocional como física (MELO et al., 2016).

De acordo com Harsraj (2014), a média mundial de uso dos smartphones é de duas a quatro horas 
por dia para checar o Facebook, ler notícias, postar uma foto no Instagram ou bater papo pelo WhatsApp, somando mais de 700 a 1,400 mil horas por ano de estresse excessivo sobre a coluna, tempo que pode ser ainda maior em universitários.

Segundo os resultados deste estudo, $27 \%(n=10)$ das tecnologias são utilizadas para fins de conexão em redes sociais, $5 \%(n=2)$ utilizam para outros fins e a grande parte dos estudantes, $68 \%(n=25)$, não utilizam as tecnologias em jogos, o que supõe que, maior parte dos estudantes entrevistados utiliza para fins de estudos. Segundo estudo realizado por Picon et al. (2015) o grande problema em questão, diz respeito à forma como os adolescentes fazem uso destas, além do isolamento social, outro problema que está relacionado é ao uso excessivo.

Em relação a dor ou desconforto, os dados coletados apontam que, 76\% ( $n=28)$ dos estudantes apresentam dor ou desconforto nos últimos doze meses, em diferentes regiões anatômicas com sintomas dolorosos, na qual, se destacam dor na coluna cervical, que resultou em 51\%, 16\% em ambos os ombros, punho/mão direita resultando em $27 \%$, ambos punhos/mãos $11 \%$ e $41 \%$ apresentam dor/desconforto na região lombar. Os dados coletados para este estudo corroboram com pesquisas já realizadas, como o estudo realizado por Perkiö et al. (2006) na qual, 48\% dos adultos/jovens apresentavam dor na cervical/ombro, $32 \%$ em braços e ombros, $19 \%$ dor em pulsos e nos dedos, $28 \%$ coluna lombar e $24 \%$ em quadris e nas pernas.

Saueressig et al. (2015) em seu estudo, relata que a dependência dessas tecnologias, vem causando uma grande preocupação, pois são utilizados demasiadamente. Tal dependência está gerando sobrecarga mental, excesso de peso, sedentarismo, dor musculoesquelética e diminuição da duração do sono, induzindo agressividade, irritabilidade, cansaço, além da cefaleia, que são os sintomas entre os adolescentes. Posturas inadequadas por longo período de tempo, normalmente adotadas durante o uso excessivo, pode justificar a presença de alguns desses sintomas.

Corroborando esses resultados, Hakala et al. (2012) também verificaram que o uso das tecnologias está associado com presença de dor na região cervical, entretanto com um tempo médio de uso de duas horas diárias. Outro estudo (TORSHEIM et al., 2010) apontou que quanto maior for o tempo exposto às tecnologias (smartphone e notebook), maior as chances do desenvolvimento de dores na coluna vertebral. Zapata et al. (2006) não encontrou associação entre dor e o uso das tecnologias, o que diverge dos resultados encontrados no presente estudo.

Diante dos fatos citados acima, observa-se que a falta de pausas durante o uso dessas tecnologias, somada ao esforço excessivo de atividades, posições inapropriadas por longos períodos de utilização acumulam lentamente prejuízos para o corpo e podem provocar lesões em várias regiões, principalmente nos membros superiores, tais como mãos, punhos, braços, ombros e pescoço, cabeça e a coluna vertebral principalmente a coluna lombar, as quais acabam se transformando em uma condição grave e muito dolorosa que não se apresenta de imediato. São dores que vêm ao longo do tempo e, que aumentam o risco se o usuário os utilizar por muitas horas seguidas e diariamente (COOPERA et al., 2004).

Posturas anteriorizadas sustentadas pela cervical podem ocasionar fadiga dolorosa no levantador 
da escápula, nos romboides e na porção inferior do trapézio, uma condição conhecida como síndrome do pescoço cansado. Os músculos traumatizados podem causar dor que, por sua vez, resulta na restrição de movimentos. Os indivíduos com essas anormalidades posturais podem sentir dor miofascial que ocasiona dor em zona referida. Durante esse processo ocorre um relaxamento anormal em alguns músculos, com encurtamento, alongamento e perda do tônus em outros, resultando em lesão e disfunção articular (HAMILL et al., 2012).

Além da presença e localização da dor, existe a recomendação da avaliação de sua intensidade. Em relação a intensidade da dor encontrados nos estudantes, $22 \%(n=10)$ referem dor 3 classificada como dor leve, $18 \%(n=8)$ referem dor 10 classificada como grave, 16\% $(n=7)$ referem dor 5 classificada como moderada, esses resultados podem ser advindos do tempo prolongado em posições estáticas, resultando em dores na coluna vertebral, como consta nos resultados obtidos neste estudo, onde $50 \%$ dos estudantes referem dor se permanecer em uma mesma postura em demasia, $36 \%$ ao realizar movimentos repetitivos.

Com isso, pode-se inferir que é alto o tempo despendido do uso de notebook e smartphone pelos estudantes avaliados; o uso desses equipamentos está sendo utilizados de forma exagerada e inadequada, o que de fato vem sendo um fator preocupante para a saúde desses usuários, diante disso observou-se que $(n=25)$ referem presença de dor, sendo que $(n=15)$ classificam esta percepção de dor como moderada e grave, esses resultados podem ser advindos do tempo prolongado em posições estáticas, resultando em dores na coluna vertebral, como consta nos resultados obtidos neste estudo, onde $50 \%(n=25)$ dos estudantes referem dor se permanecer em uma mesma postura em demasia, e $36 \%(n=18)$ ao realizar movimentos repetitivos. Nisso, observa-se que quanto maior o tempo de uso diário destas tecnologias, maior a incidência de dores musculoesqueléticas.

Contudo, observa-se que o estudo de Hakala et al. (2012) foi o único que avaliou a associação do uso de computadores com a localização e intensidade dos sintomas dolorosos na coluna vertebral de adolescentes. Estudo realizado por Silva et al. (2012) optaram por não avaliar a intensidade da dor, pois os dados deveriam ser analisados com cautela, pois acreditam que o corte transversal não permite inferir a possível causalidade, bem como a avaliação por autorrelato pode apresentar viés de memória, podendo comprometer os resultados. Por outro lado, outro estudo realizado com adolescentes finlandeses (HAKALA et al., 2010), sugere que os sintomas musculoesqueléticos que causam dor moderada a grave são comuns entre usuários das tecnologias.

\section{CONCLUSÕES}

O estudo demostrou alta prevalência de dor musculoesquelética nos entrevistados, evidenciados pela longa permanência na utilização destas tecnologias, demonstrando que o uso excessivo destes dispositivos incide sobre a percepção dolorosa nas seguintes regiões: coluna cervical, ombros e coluna lombar. Nisto, observa-se que quanto maior o tempo de uso diário destas tecnologias, maior a incidência de dores musculoesqueléticas. Assim, é necessária a conscientização do uso destas tecnologias, de forma cautelar, a fim de prevenir o desenvolvimento dos índices de queixas relacionadas a esse uso 
indiscriminado.

\section{REFERÊNCIAS}

ANDREGHETTO, R.. Uso excessivo de telefones e computadores pode causar lesões graves, 2015.

GUAN, X.; FAN, G,; CHEN, Z.; ZENG, Y.; ZHANG, H.; HU, A.; GU, G.; WU, X.; GU, X.; HE, S.. Gender difference in mobile phone use and the impact of digital device exposure on neck posture. Ergonomics, v.59, n.11, p.1453-1461, 2016.

COOPERA, K. L. N.; SOMMERICHA, C. M.; MIRKAA, G. A.. College students and computers: assessment of usage patterns and musculoskeletal discomfort. Work: A Journal of Prevention, Assessment and Rehabilitation, v.32, n.3, p.285-98, 2009.

DAS, B.. Assessment of ergonomical and occupational health related problems among VDT workers of West Bengal, India. AJMS, p.26-31, 2014.

DICKINSON, K.; CAMPION A.; FOSTER, S.; NEWMAN, A.; O'ROURKE, M.; THOMAS, P.. Questionnaire development: an examination of the Nordic Musculoskeletal Questionnaire. Applied ergonomics, v.23, p.197-201, 1992.

HAKALA, P. T., SAARNI, L. A., KETOLA, R. L., RAHKOLA, E. T.; SALMINEN, J. J.; RIMPELÄ, A. T.. Computer-associated health complaints and sources of ergonomic instructions in computer-related issues among finnish adolescents: a crosssectional study. BMC Public Health, v.10, 2010.

HAKALA, P. T.; SAARNI, L. A.; PUNAMAKI, R. L; WALLENIUS M. A; NYGÂRD, C. H.; RIMPELA, A. H.. Musculoskeletal symptoms and computer use among finnish adolescents pain intensity and inconvenience to everyday life: a crosssectional study. BMC Musculoskelet Disord, v.10, 2012.

HAMILL, J.; KNUTZEN, K. M.. Bases Biomecânicas do Movimento Humano. São Paulo: Manole, 2012.

HANSRA, J.; KENNETH, K.. Assessment of Stresses in the Cervical Spine Caused by Posture and Position of the Head. Urgical Technology International, p.277-279, 2014.

hoffMANN, J.; TEODOROSKI, R.. A Eficácia da Pompage, na coluna cervical, no tratamento da cefaléia do tipo tensional. Universidade do Sul de Santa Catarina, 2009.

KHAN, R.; SURTI, A.; REHMAN, R.; A. L, U.. Knowledge and practices of ergonomics in computer users. JPMA, v.62, n, 3, 2012.

KING, S.; CHAMBERS, C. T; HUQUET, A.; MACNEVIN, R. C; MCGRATH, P. J; PARKER, L.; PONTES, H.; PATRÃO, I.. Estudo Exploratório Sobre as Motivações Percebidas no uso Excessivo da Internet em Adolescentes e Jovens Adultos. Psychology, Community \& Health, v.3, n.2, 2014.

LUCENA, J.; CHENG, L.; CAVALCANTE, T.; SILVA, V; FARIAS, J. Prevalência de tempo excessivo de tela e fatores associados em adolescentes. Revista Paulista Pediatria, v.33, n.4, p.407-414, 2015.

MAGEE, D.. Avaliação Musculoesquelética. 4 ed. São Paulo: 2005.
MACDONALD, A.; J.. The epidemiology of chronic pain in children and adolescents revisited: a systematic review. Pain, v.152, n.12, 2011.

MARTINEZ, J.; GRASSI, D.; MARQUES, L.. Análise da aplicabilidade de três instrumentos de avaliação de dor em distintas unidades de atendimento: ambulatório, enfermaria e urgência. Revista Brasileira Reumatologica, v.51, n.4, p.299-308, 2011.

MELO, G.. A Nomofobia entre crianças e adolescentes. In: CONGRESSO INTERNACIONAL DE PSICOLOGIA DA CRIANÇA E DO ADOLESCENTE, 7. Anais. Universidade Lusíada, 2016.

PARK, J.; KIM, K.; KIM, N.; CHOI, I.; LEE, S.; TAK, S.; YIM, S.. A Comparison of Cervical Flexion, Pain, and Clinical Depression in Frequency of Smartphone Use. International Journal of Bio-Science and Bio-Technology, v.7, p.183-190, 2015.

PERKIÖ-MÄKELÄ M., HIRVONEN, M.; ELO, A.; ERVASTI, J.; HUUHTANEN, P.; KANDOLIN, I.. Work and health interview study. Report of tables. Helsinki, Finland: Finnish Institute of Occupational Health, 2006.

PICON, F.; KARAM, R.; BREDA, V.; RESTANO, A.; SILVEIRA, A.; SPRITZER, D.. Precisamos falar sobre tecnologia: caracterizando clinicamente os subtipos de dependência de tecnologia. Rev. Bras. Psicoterapia, v.17, n.2, p.44-60, 2015.

PIMENTA, C.; MATTOS, A.; JACOBSEN, M.. Questionário de dor McGill: proposta de adaptação para a língua portuguesa. Rev. Esc. Enferm., v.30, n.3, p.473-483, 1996.

SAUERESSIG, I.; XAVIER, M.; OLIVEIRA, V.; PITANGUI, A.; ARAÚJO, R.. Primary headaches among adolescents and their association with excessive computer use. Rev. Dor, v.16, n.4, p.8-244, 2015.

SILVA, G.; PITANGUI, A.; XAVIER, M.; CORREIA-JÚNIOR, M.; ARAÚJO, R.. Prevalência de dor musculoesquelética em adolescentes e associação com uso de computador e jogos eletrônicos. Jornal de Pediatria, v.92, n.2, 2016.

SOUZA, J.; GOBBY, M.. Geração Digital: uma reflexão sobre as relações da "juventude digital" e os campos da comunicação e da cultura. Revista Gemini, v.2, p.129-145, 2014.

TAPSCOTT, D.. A hora da geração digital: como jovens que cresceram usando a internet estão mudando tudo, das empresas aos governos. Rio de Janeiro: Agir Negócios, 2010.

TEIXEIRA, M.; YENG, L.; KAZIYAMA, H.. Dor, síndrome dolorosa, miofascial e dor, músculo-esquelética. São Paulo, 2006.

TELECO. Estatísticas de Celulares no Brasil, 2016.

TORSHEIM, T.; ERIKSSON, L.; SCHNOHR, C. W.; HANSEN, F.; BJARNASON, T.; VALI-MAA, R.. Screen-based activities and physical complaints among adolescents from the Nordic countries. BMC Public Health, 2010. 
ZAPATA, A. L.; MORAES, A. J.; LEONE, C.; DORIA-FILHO, U.; SILVA, C. A.. Pain and musculoskeletal pain syndromes related to computer and video game use in adolescents. Eur J Pediatr, v.165, n.6, 2006.
YOUNG, K. S.; RODGERS, R. C.. The relationship between depression and Internet addiction. Cyber Psychol Behav., v.1, n.1, 8-25, 1998

A CBPC - Companhia Brasileira de Produção Científica (CNPJ: 11.221.422/0001-03) detém os direitos materiais desta publicação. Os direitos referem-se à publicação do trabalho em qualquer parte do mundo, incluindo os direitos às renovações, expansões e disseminações da contribuição, bem como outros direitos subsidiários. Todos os trabalhos publicados eletronicamente poderão posteriormente ser publicados em coletâneas impressas sob coordenação da Sustenere Publishing, da Companhia Brasileira de Produção Científica e seus parceiros autorizados. Os (as) autores (as) preservam os direitos autorais, mas não têm permissão para a publicação da contribuição em outro meio, impresso ou digital, em português ou em tradução. 